\title{
Expression , Purification, and Characterization of the Recombinant, Two-component, Response Regulator ArIr From Fusobacterium Nucleatum
}

Ruochen Fan ( $\square$ fanruochen@mail.dlut.edu.cn )

Dalian University of Technology; Dalian Minzu University

Zhuting Li

Dalian Minzu University: Dalian Nationalities University

Chunshan Quan

Dalian Minzu University: Dalian Nationalities University

Lulu Wang

Dalian University of Technology; Dalian Minzu University

Xian Shi

Dalian Minzu University: Dalian Nationalities University

Xuqiang Zhang

Dalian Minzu University: Dalian Nationalities University

Yuesheng Dong

Dalian University of Technology

\section{Research Article}

Keywords: two-component system, Fusobacterium nucleatum, protein expression and purification, protein interaction

Posted Date: June 7th, 2021

DOI: https://doi.org/10.21203/rs.3.rs-571272/v1

License: (c) (1) This work is licensed under a Creative Commons Attribution 4.0 International License.

Read Full License

Version of Record: A version of this preprint was published at Applied Biochemistry and Biotechnology on January 14th, 2022. See the published version at https://doi.org/10.1007/s12010-021-03785-5. 


\section{Abstract}

Fusobacterium nucleatum is associated with the incidence and development of multiple diseases, such as periodontitis and colorectal cancer (CRC). Till now, studies have proved only a few proteins to be associated with such pathogenic diseases. The two-component system is one of the most prevalent forms of bacterial signal transduction related to intestinal diseases. Here we report a novel, recombinant, two-component, response-regulator protein ArIR from the genome of $F$. nucleatum strain ATCC 25586 . We optimized the expression and purification conditions of ArIR; in addition, we characterized the interaction of this response regulator protein to the corresponding histidine kinase and DNA sequence. The fulllength ArIR was successfully expressed in six of the E. coli host strains. However, optimum expression conditions of ArIR were present only in E. coli strain BL21 Condon plus (DE3) RIL that was later induced with isopropyl $\beta$-D-1-thiogalactopyranoside (IPTG) for $8 \mathrm{~h}$ at $25^{\circ} \mathrm{C}$. The SDS-PAGE analysis revealed the molecular weight of the recombinant protein as $27.3 \mathrm{kDa}$ and approximately $90 \%$ purity after gel filtration chromatography. ArlR was biologically active after its purification. Therefore, it accepted the corresponding phosphorylated histidine-kinase phosphate group and bound to the analogous DNA sequence. The binding constant between ArIR and the corresponding histidine kinase is $1.28 \mu \mathrm{M}$, while the binding constant between ArlR and the bound DNA sequence is $37.5 \mu \mathrm{M}$. Altogether, these results illustrate an effective expression and purification method for the novel two-component system protein ArlR.

\section{Introduction}

Fusobacterium nucleatum is a typical, opportunistic, and pathogenic gram-negative bacteria related to the incidence and development of diseases such as periodontitis, head and neck cancer, poor pregnancy, and colorectal cancer (CRC) [1-4]. F. nucleatum can invade endothelial cells and adhere to several mammalian cells, including murine lymphocytes and macrophages, and human oral epithelial cells to participate in colonization and evade host defenses $[5,6]$. Noticeably, F. nucleatum has been reported to be present in a high abundance in CRC cells [7]. Many studies have shown that $F$. nucleatum plays a significant role in increasing the proliferation and migration of CRC cells; because of it forming a proinflammatory micro-environment suitable for the development of CRC and enhancing the drug resistance of CRC cells [8-10]. In dental biofilms, F. nucleatum through periodontitis-pathogen accretion connects the early colonizing commensals and late pathogenic colonizers. Also, F. nucleatum functions as a connector between the primary and secondary colonizing organisms. Therefore, F. nucleatum is commonly known as a "bridge organism" $[11,12]$.

A two-component system (TCS) is a form of signal-transduction system that mainly exists in microorganisms and plants [13]. Bacterial signal-transduction systems mainly consist of two proteins: histidine-kinase and transcription protein. Bacterial signal-transduction systems use kinases with extracellular or periplasmic sensing domains for transferring the phosphate groups to its corresponding response regulator protein; thus, altering the levels of gene expression [14-21]. TCSs are involved in bacterial pathogenicity, biofilm formation, and physiological responses to osmotic changes in various 
bacteria [14]. Recently, many studies have found that the TCS is related to intestinal diseases [22-28]. Wakimoto S. et al. (2013) proved that phosphate regulon transcriptional regulatory protein (PhoB) was necessary for Bacteroides fragilis survival in peritoneal abscesses [26]. Likewise, Massmig M. et al. (2020) suggested that the CntAB two-component system in the gut micobiome catalyzes the oxidative cleavage of L-carnitine into TMA related to cardiovascular diseases [23]. Another significant characteristic of the two-component system is its ability to promote colonization of bacteria in the mammalian intestine; for example, FusKR TCS is required for the robust colonization of enterohemorrhagic E. coli in the mammalian intestine [29]. The CpxRA TCS of Salmonella enterica plays a significant role in gut colonization in Salmonella-induced colitis [30]. Moreover, the two-component system also affects the metabolic response of the intestinal bacteria [31-33]. Sonnenburg ED. et al. (2006) demonstrated that hybrid TCSBT3172 functions as a metabolic reaction center by coupling the nutrient-sensing to the dynamic regulation of monosaccharide metabolism [32], and Liu M. et al. (2019) proved that CitAB TCS in Vibrio cholerae contributes to the anaerobic citrate fermentation [31].

TCSs are potential targets for antimicrobial drug design. Since the histidine phosphorylation of TCS in bacteria differs from serine/tyrosine/threonine phosphorylation of signaling systems in mammalian cells, TCS inhibitors may exert less toxicity in the host $[34,35]$. TCSs have been well-studied in several pathogenic bacteria such as Escherichia coli and Pseudomonas aeruginosa; however, they have not been well-studied in F. nucleatum. In this study, we have analyzed the two-component system of $F$. nucleatum ATCC 25586, one of the recently sequenced genome strains, which further enhances our knowledge and understanding of its physiological function and pathogenic mechanism of virulence.

In summary, this study provides a method for the expression, purification, and characterization of the response regulator (RR), TCS protein ArIR. This paper is the first to report the purification and characterization of the TCS-RR protein ArIR in F. nucleatum.

\section{Methods}

\section{Bacterial strains, plasmids, enzymes, and chemicals}

We purchased F. nucleatum strain ATCC 25586 from American Type Culture Collection (ATCC). The E. coli strains (DH5a, BL21 (DE3), BL21-Condon Plus (DE3) RIL, BL21 (DE3) pLysS, Tuner (DE3), C43 (DE3), Transetta (DE3)), and the plasmid $p E T-28 a$ were in the preserved state in our laboratory. Also, we purchased the HS DNA polymerase and restriction enzyme from Takara BioEngineering (Dalian, China). DNA gel extraction and plasmid purification kits were purchased from Axygen (corning, America). Chemicals used, such as kanamycin, chloramphenicol, and $\beta$-D-1-thiogalactopyranoside (IPTG), were of analytical grade unless otherwise stated.

\section{Bioinformatics Analysis}

The complete genome sequence of $F$. nucleatum ATCC 25586 (NP_603483.1) was obtained in FASTA format from the NCBI database (https://www.ncbi.nlm.nih.gov/). The Stockholm format files from the 
conserved protein domain family HATPase_c (Pfam02518) of histidine-kinase (HK) and from the conserved domain family Response_reg (Pfam00072) of RR protein was downloaded from the Pfam database (http://pfam.xfam.org/). In HMMER 2.0 software, the command hmmbuild (see Table 1 for commands) was used to construct the hidden Markov models for HATPase_c and Response_reg, which finds out the HKs and RRs with HATPase_c and Response_reg domains. Subsequently, comparing the obtained results with those on the MiST website provides a comprehensive classification of the signaltransduction systems [36]. Finally, we stated three points to determine a pair of two-component signal systems: 1) histidine kinase catalytic domain HATPase_c must be located at the HK C-terminus; 2) the upstream of HATPase_c should have the phosphate receptor domain HisKA; 3) N-terminus of RR should be the phosphate receptor in the target TCSs. Multiple sequence alignments were performed using ClustalX and ESPript [37]. The molecular architecture of the TCS response regulator proteins and ArIR was determined by SMART and Pfam [38]. The structure was predicted from Phyre ${ }^{2}$ [39].

\section{Construction of the recombinant plasmid}

Using the restriction-free (RF) cloning method, we constructed the $p E T-28 a$-arlR plasmid. The RFcloning.org web server was used to design primers F1 ( ${ }^{5}$ - CAGCCATCATCATCATCATCACAGCAGC ATGTTATTATTTTCTTGGGTGAGG ${ }^{-3}$ ', where the sequence in italics represents arlR gene-specific region) and R1 ( ${ }^{\prime}$ - GGAGCTCGAATTCGGATCCGCG TTAATCCTCTTTATATTGAAATATATAG ${ }^{-3}$ ', where sequence in italics represent the arIR gene-specific region) complementary to insert and vector [40]. The arlRmegaprimers (the first polymerase chain reaction (PCR)) were amplified by PCR, using the primers F1 and R1 from the genomic DNA of strain ATCC 25586 of F. nucleatum. The PCR product was collected by the Axygen Prep DNA Gel Extraction Kit and then inserted into pET-28a plasmid by the secondary PCR reaction (100 ng megaprimers, $200 \mu \mathrm{M}$ dNTP, $25 \mathrm{ng} p E T-28 a$ vector, and 1 U HS DNA Polymerase). The

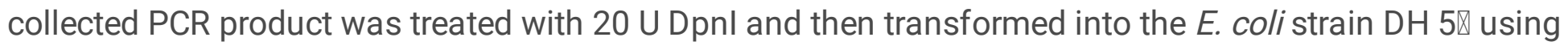
electroporation.

The cytoplasmic domain of histidine kinase (ar/SC) was amplified with the primers F2 $\left(5^{\prime}\right.$ CAGCCATCATCATCATCATCACAGCAGC GATAAATTTAAAAATTCACTTG ${ }^{-3}{ }^{3}$, where sequence in italics represents arlSC gene-specific region) and R2 ( $5^{\prime}$ - GGAGCTCGAATTCGGATCCGCG TTAAAATAGTAGTGTTATTTTTGTTCCC ${ }^{-3}$ ', where sequence in italics represents arlSC gene-specific region). The cloning steps of arlSC were carried out as described above for ArlR.

\section{Expression of the recombinant ArIR}

Six different E. coli host strains (BL21 (DE3), BL21-Condon plus (DE3) RIL, BL21 (DE3) pLysS, Tuner (DE3), C43 (DE3), Transetta (DE3)) were used to express ArlR. We selected a positive transformant from each of the host strains and cultured it overnight at $37^{\circ} \mathrm{C}$ in $20 \mathrm{~mL}$ Luria-Bertani (LB) medium supplemented with the corresponding antibiotics; next, the liquid culture medium was transformed into $50 \mathrm{~mL}$ LB medium with $1 \%$ culture inoculation and cultivated at $37^{\circ} \mathrm{C}$ until the $\mathrm{OD}_{600}$ reached $0.6-0.8$. Subsequently, the protein expression was induced by the addition of $0.5 \mathrm{mM} \mathrm{IPTG}$ at different intervals of 
time $(8 \mathrm{~h}, 20 \mathrm{~h})$ and temperatures $\left(16^{\circ} \mathrm{C}, 25^{\circ} \mathrm{C}\right)$. The cells were harvested by centrifugation, resuspended in $1 \mathrm{~mL}$ buffer $\mathrm{A}(20 \mathrm{mM}$ Tris- $\mathrm{HCl} \mathrm{pH} \mathrm{8.0,150} \mathrm{mM} \mathrm{NaCl})$, and disrupted by sonication for $5 \mathrm{~min}$ on ice. The lysate was then centrifuged at $18,200 \times \mathrm{g}$ for $10 \mathrm{~min}$ at $4{ }^{\circ} \mathrm{C}$. The soluble and insoluble proteins were detected by sodium dodecyl sulfate-polyacrylamide gel electrophoresis (SDS-PAGE).

\section{Purification of the recombinant protein}

The $E$. coli host strain with the highest protein expression was selected for large-scale protein expression through the subsequent purification steps. Five grams of wet cells, harvested by centrifugation, were resuspended in $35 \mathrm{~mL}$ of buffer $A$. Aliquots of $1 \mathrm{~mL}$ were taken from the $35 \mathrm{~mL}$ resuspended buffer. Each aliquot was centrifuged for precipitation. Cells were resuspended in buffer $A$ containing different additives (5\% glycerol, $50 \mathrm{mM}$ L-Arg, $50 \mathrm{mM}$ betaine, 1\% Triton X-100, $50 \mathrm{mM}$ L-Arg and $50 \mathrm{mM} \mathrm{L-Glu}$ ) separately and, respectively. The cells were then disrupted by sonication and analyzed by $15 \%$ SDS-PAGE, which revealed buffer $\mathrm{A}$ with $5 \%$ glycerol to be the most suitable buffer. The crude extracts were precipitated by centrifugation at $18,200 \times \mathrm{g}$ for $45 \mathrm{~min}$ at $4{ }^{\circ} \mathrm{C}$. The soluble supernatant of the lysate was mixed with $5 \mathrm{~mL}$ Ni-NTA affinity resin (GE Healthcare, USA), and the solution was incubated for $30 \mathrm{~min}$ in a rotating shaker at $4{ }^{\circ} \mathrm{C}$. We further loaded the resulting slurry onto a $50 \mathrm{~mL}$ column, where the resin was washed away with buffer B (20 mM Tris- $\mathrm{HCl}, \mathrm{pH} 8.0,150 \mathrm{mM} \mathrm{NaCl}, 5 \%$ glycerol, $20 \mathrm{mM}$ imidazole). The target protein was eluted from the column by buffer C $(20 \mathrm{mM}$ Tris-HCl, pH 8.0, $150 \mathrm{mM} \mathrm{NaCl}, 5 \%$ glycerol, $250 \mathrm{mM}$ imidazole). The eluted protein was concentrated and loaded onto the HiLoad Superdex 200 26/60 column (GE Healthcare, USA) and was equilibrated with buffer A containing $5 \%$ glycerol. We used $15 \%$ SDS-PAGE and Coomassie staining to analyze the samples obtained from each purification step. The expression and purification of ArISC were carried out as described above for ArlR.

\section{Circular dichroism spectroscopy}

Circular dichroism (CD) spectroscopy was performed on a Chirascan ${ }^{\mathrm{TM}}$ spectrometer (Applied Photophysics Ltd) with a $0.1 \mathrm{~cm}$ path length of the quartz cuvette. The samples were prepared using buffer A (20 mM Tris-HCl, pH 8.0, $150 \mathrm{mM} \mathrm{NaCl}$ ) and $0.2 \mathrm{mg} / \mathrm{mL}$ ArlR. The sample was $200 \mu \mathrm{L}$ with $1 \mathrm{~nm}$ bandwidth length, $185-260 \mathrm{~nm}$ scanning range, and $0.5 \mathrm{~s}$ time-per-point. The spectrum of the target protein at $191-260 \mathrm{~nm}$ was obtained over a range of temperatures starting from $20^{\circ} \mathrm{C}$ and incrementally increasing to $94^{\circ} \mathrm{C}$, which we used to measure the ArlR melting temperature (Tm). Ultimately, the Tm value was calculated by the Global 3 software, while the secondary structure was visualized and analyzed using the Deconvolution software that comes with the instrument.

\section{Autophosphorylation and phosphoryl transfer assays}

Purified ArlSC was pre-equilibrated with the phosphorylation buffer $(20 \mathrm{mM}$ Tris- $\mathrm{HCl}$ at pH 8.0, $50 \mathrm{mM}$ $\mathrm{KCl}, 5 \mathrm{mM} \mathrm{MgCl}$ ) in a final volume of $100 \mu \mathrm{L}$. Subsequently, an autophosphorylation reaction was initiated by adding the aliquots of ATP to a final concentration of $10 \mu \mathrm{M}$ and lasting for $30 \mathrm{~min}$ at $25^{\circ} \mathrm{C}$. The intrinsic kinase activity of ArISC was measured using the Promega Kinase-Glo Luminescent Kinase Assay Kit. As for the phosphoryl transfer assays, the purified ArlR was added to the phosphorylated ArlSC 
to initiate phosphotransfer reaction in the phosphorylation buffer with $10 \mu \mathrm{M}$ ATP for 10 min at $25^{\circ} \mathrm{C}$. Lastly, the persistent ATP was measured by the Promega Kinase-Glo Luminescent Kit.

\section{Microscale thermophoresis (MST) assay}

As previously reported [41], the affinity of ArIR and ArISC was determined by MST using Monolith NT.115 (NanoTemper Technologies, Germany). Briefly, ArISC was labeled with NHS fluorescent dye and centrifuged at $18,200^{\circ} \mathrm{g}$ for $10 \mathrm{~min}$ to eliminate precipitation. A 16 step 1:1 (v/v) ArIR serial stock, with twofold dilution, was prepared with the MST buffer (50 mM Tris- $\mathrm{HCl}, \mathrm{pH} 8.0,150 \mathrm{mM} \mathrm{NaCl}, 10 \mathrm{mM} \mathrm{MgCl}$, $0.05 \%$ Tween-20), such that each dilution step reduced the protein concentration by $50 \%$. Equal volumes of the labeled ArISC and unlabeled ArIR were mixed, incubated for $5 \mathrm{~min}$ at room temperature, and added to capillaries for measurement. The data were analyzed using NanoTemper Analysis software.

According to the protocol as described previously [42], the binding of ArlR to its promoter was determined by MST using Monolith NT.115 (NanoTemper Technologies, Germany).Briefly, the promoter of ArIR was amplified with primers of F2 (Cy5 - 5' - CCTCACCCAAGAAAATAATAATAAC $\left.{ }^{-3}\right)$ and R2 ( ${ }^{\prime}$ CCAAATAAGGCATAAGAGAGC ${ }^{-3}$ '). A 16 step 1:1 (v/v) serial ArlR stock, with twofold dilution, was prepared with the MST buffer (50 mM Tris- $\mathrm{HCl}$ at pH 8.0, $150 \mathrm{mM} \mathrm{NaCl}, 10 \mathrm{mM} \mathrm{MgCl}$, 0.05\% Tween-20). Equal volumes of the labeled DNA and unlabeled protein solutions were mixed and incubated for $5 \mathrm{~min}$ at room temperature. Lastly, the samples were loaded into silica capillaries and measured by MST instrument.

\section{Results}

\section{Two-component system in F. nucleatum strain ATCC 25586}

The two-component system of F. nucleatum strain ATCC 25586 was searched by the software HMMER, and compare with the results on the website MiST. Four pairs of the typical TCSs identified were CzcRS, ArIRS, LytRS, and YesNM, respectively. The response regulator (RR) proteins in F. nucleatum strain ATCC 25586 contained a conserved receiver domain and a variable effector domain (Fig. 1a). Previous studies have reported that the response regulatory ArlR contributes to adhesion, autolysis, and multidrug resistance $[43,44]$. The alignment analysis between the ArIR in F. nucleatum ATCC 25586 and other reported response regulation ArlR showed a high sequence similarity. By comparing with the sequence of the ArIR protein in Staphylococcus aureus, it can be said that the conserved aspartate phosphate group receptor in the 61st residue is most likely the phosphate acceptor site (Fig. 1d). These results indicated ArIR protein in F. nucleatum strain ATCC 25586 as a typical response regulatory protein in the twocomponent system.

ArlR is a member of the OmpR/PhoB superfamily that consists of a receiver domain and an effector domain. However, the domain predicted from Pfam (Fig. 1b) and the structure predicted from the Phyre ${ }^{2}$ (Fig. 1c) shows an extended flexible region between these two domains. Also, the gene of the ArlR is composed of a large amount of AT base pairs. Therefore, we predict that these conditions may cause 
certain difficulties in the expression or purification of ArIR, so we optimized its expression and purification conditions.

\section{Expression and purification of the recombinant ArIR}

The arlR gene from F. nucleatum strain ATCC 25586 was inserted into the $p E T-28 a$ plasmid to create a recombinant plasmid and then separately transformed into the six different host strains. The expression of ArIR protein varied significantly with the varying host strains after inducing $0.5 \mathrm{mM} \mathrm{IPTG}$ for $8 \mathrm{~h}$ or 20 $\mathrm{h}$, at $16^{\circ} \mathrm{C}$ or $25^{\circ} \mathrm{C}$. Host strains had the most significant impact on ArlR expression; however, the best expression conditions were present in E. coli host strain BL21 Condon plus (DE3) RIL induced with 0.5 $\mathrm{mM}$ IPTG for $8 \mathrm{~h}$ at $25^{\circ} \mathrm{C}$ (Fig. 2a). Several additives such as glycerin, L-arginine, $50 \mathrm{mM} \mathrm{L-Arg}$ and 50 mM L-Glu, betaine, $1 \%$ Triton X-100 were added to increase purified ArlR soluble protein. SDS-PAGE revealed that the recombinant ArIR was expressed both in the soluble and inclusion body forms; and the solubility of ArlR could increase slightly by adding $5 \%$ glycerol to the additive (Fig. 2 b).

Further, ArlR was purified by Ni-NTA affinity chromatography and gel filtration chromatography. Using NiNTA affinity chromatography, we obtained ArlR with a high concentration and purity (Fig. 3a). However, using the purification process of the gel filtration chromatography, the ArlR protein got slightly degraded (Fig. 3b). ArlR was collected into a 50-mL concentration tube after gel filtration chromatography and was concentrated to $500 \mu \mathrm{L}$ by centrifugation at $4{ }^{\circ} \mathrm{C}$. The yield of ArlR was about $10 \mathrm{mg} / \mathrm{L}$ of culture solution. The secondary structure and thermal stability of the purified ArIR were further determined by circular dichroism spectroscopy. Two minimum peaks at $208 \mathrm{~nm}$ and $222 \mathrm{~nm}$ of ArlR are the characteristic structural peaks of a-helix; thus, indicating that the purified ArIR has secondary structure-activity (Fig. 3c). Moreover, circular dichroism (CD) spectra of ArlR at temperatures $20^{\circ} \mathrm{C}-94^{\circ} \mathrm{C}$ showed the changes in the secondary structure of the protein at different temperatures (Fig. $3 \mathrm{~d}$ ). Consequently, the height of the characteristic peak of the ArIR a-helix gradually decreased with the increase in temperature, which may be due to the dissociation and the changes of the protein secondary structure. Calculations show the Tm of ArlR as $55.8^{\circ} \mathrm{C}$.

\section{The phosphotransferase activity of the phosphorylated ArISC to ArlR}

Kinase-Glo ${ }^{\text {TM }}$ Luminescent Kinase assay detected the presence of the kinase activity of cytoplasmic domain of histidine kinase (ArISC). As shown in Fig. 4a, the luminescence intensity of the reaction system gradually decreased, that is, the remaining ATP content in the kinase buffer gradually decreased with the increase in the concentration of ArISC; thus, suggesting that the ArISC protein has histidine kinase activity. Using microscale thermophoresis (MST) experiment, we investigated an interaction between the phosphorylated histidine-kinase cytoplasmic domain (P-ArISC) and its corresponding response regulator (ArlR). The outcome reveals that P-ArlSC can interact with ArlR, with high affinity ( $\mathrm{Kd}=1.28 \mu \mathrm{M}$, Fig. 5a). We further investigated the phosphotransferase activity of P-ArlSC to ArlR by adding the different concentrations of ArIR to the P-ArISC $(3 \mu \mathrm{M})$ solution containing ATP. The remaining ATP content in the kinase buffer gradually decreased with an increase in the concentration of ArlR; thus, suggesting that the P-ArISC protein has the phosphotransferase activity to ArlR (Fig. 4b). 


\section{The binding of phosphorylated ArIR to its promoter}

The response regulatory protein of the TCS can usually be in combination with its promoter to regulate its expression. To test this hypothesis, we designed a pair of primers labeled with cy- 5 and amplified approximately 300 bp sequence present between the arlR gene (Fn1260) and its upstream gene (Fn1259) in F. nucleatum strain ATCC 25586. Using MST experiment, we confirmed the interaction between P-ArIR and the corresponding DNA sequence. The intermolecular Kd value of P-ArIR and its promoter is $37.5 \mu \mathrm{M}$ (Fig. 5b).

\section{Discussion}

The ArlR is a member of the OmpR/PhoB subfamily, the largest subfamily of bacterial RRs [45]. These RRs can bind to DNA; thus, activating transcription. The TCS of ArIRS plays a significant role in grampositive bacteria, such as Staphylococcus aureus and Staphylococcus epidermidis. Bai J et al. (2019) confirmed that eliminating the ArIRS TCS in certain strains of Staphylococcus aureus may increase sensitivity to oxacillin [46]. Moreover, JN Radin et al. (2016) proved that the ArIRS TCS enhanced the proliferation of Staphylococcus aureus in the presence of calprotectin-induced manganese-starvation [47] . However, what is the function of the TCS of ArIRS in gram-negative bacteria is still unclear. We found that the ArIRS TCS of F. nucleatum is highly homologous to the ArIR TCS of Staphylococcus aureus. The ArlR was expressed and purified in vitro to investigate the function of ArlRS from F. nucleatum. Results found that ArIR is a typical RR protein which could receive a phosphoryl group from its corresponding histidine-kinase cytoplasmic ArISC through phosphoryl transfer. Therefore, this result suggests that the interaction between ArlS histidine-kinase and ArIR response regulator protein mainly relies on the cytoplasmic domain of histidine-kinase, while the transmembrane-domain of ArlS histidine-kinase may play significant roles in the sensing and transmitting of signals.

Studies have reported that different host strains of $E$. coli have different characteristics. Therefore, different host strains may be used to express different types of proteins $[48,49]$. We proposed six host strains of $E$. coli to explore the differences in the expression of the same protein in different host bacteria. The results showed that the highest expression of ArIR was in E. coli BL21-Condon plus (DE3) RIL host strain. The genotype of E. coli BL21-Condon plus (DE3) RIL is derived from E. coli BL21 (DE3) and possesses rare tRNAs for expressing AT-rich genomes [49]. The genome sequence of $F$. nucleatum strain ATCC 25586 had $73 \%$ more AT base [50] , and the AT content of the arlR gene in the strain was $74.3 \%$. Therefore, we suggest a preferential selection of E. coli BL21-Condon plus (DE3) RIL host strain during the expression of proteins in F. nucleatum. During protein purification, the purpose of adding $5 \%$ glycerol to the buffer is that besides increasing the solubility of ArlR protein, it can effectively prevent protein degradation. In addition, we recommend using a smaller gel filtration column and a faster flow rate, which can significantly reduce the degradation and improve the purity of ArIR.

\section{Conclusion}


Altogether, we identified four groups of TCS in F. nucleatum strain ATCC 25586 and successfully cloned the arlR gene into $p E T-28 a$ by restriction-free (RF) cloning method. We obtained ArlR with a purity greater than $90 \%$ and concentration above $15 \mathrm{mg} / \mathrm{mL}$ by using the optimized expression and purification conditions. In addition we proved that the purified ArIR RR protein could receive phosphoryl group from its corresponding HK cytoplasmic ArISC, and the phosphorylated ArlR could binds to its promoter. These results also suggest that one should preferentially select $E$. coli strain BL21-Condon plus (DE3) RIL as the host bacteria during the expression of proteins from Fusobacterium nucleatum.

\section{Declarations}

\section{Funding}

This work was supported by the National Natural Science Foundation of China (Grant No. 21272031) and the Fundamental Research Funds for the Central Universities (Grant No. wd01190).

\section{Competing interests}

The authors declare that they have no competing interests

\section{Availabilityofdataandmaterials}

The datasets used and analyzed during the current study are available from the corresponding author on reasonable request.

\section{Code availability}

Not applicable

\section{Authors' contributions}

Ruochen Fan: Formal analysis, Writing - original draft, conducted experiments, acquired data, analyzed data and wrote the paper. Zhuting Li: conducted experiments and acquired data. Xian Shi: conducted experiments and acquired data. Lulu Wang: conducted experiments and acquired data. Xuqiang Zhang: conducted experiments and acquired data. Chunshan Quan: Funding acquisition. Yuesheng Dong: Funding acquisition.

\section{Ethics approval and consent to participate}

Not applicable

\section{Consent for publication}

Not applicable 


\section{References}

1. 1. Liu $Y$, Baba $Y$, Ishimoto $T$, Iwatsuki $M$, Hiyoshi $Y$, Miyamoto $Y$, et al. Progress in characterizing the linkage between Fusobacterium nucleatum and gastrointestinal cancer. Journal of gastroenterology. 2019;54(1):33-41.

2. 2. Luo $K$, Zhang $Y, X v C$, Ji J, Lou G, Guo X, et al. Fusobacterium nucleatum, the communication with colorectal cancer. Biomedicine \& Pharmacotherapy. 2019;116:108988.

3. 3. Bronzato JD, Bomfim RA, Edwards DH, Crouch D, Hector MP, Gomes BP. Detection of Fusobacterium in oral and head and neck cancer samples: A systematic review and meta-analysis. Archives of oral biology. 2020;112:104669.

4. 4. Wang X, Buhimschi CS, Temoin S, Bhandari V, Han YW, Buhimschi IA. Comparative microbial analysis of paired amniotic fluid and cord blood from pregnancies complicated by preterm birth and early-onset neonatal sepsis. PloS one. 2013;8(2):e56131.

5. 5. Weiss E, Shaniztki B, Dotan M, Ganeshkumar N, Kolenbrander P, Metzger Z. Attachment of Fusobacterium nucleatum PK1594 to mammalian cells and its coaggregation with periodontopathogenic bacteria are mediated by the same galactose-binding adhesin. Oral microbiology and immunology. 2000;15(6):371-7.

6. 6. Han YW, Redline RW, Li M, Yin L, Hill GB, McCormick TS. Fusobacterium nucleatum induces premature and term stillbirths in pregnant mice: implication of oral bacteria in preterm birth. Infection and immunity. 2004;72(4):2272-9.

7. 7. Kostic AD, Chun E, Robertson L, Glickman JN, Gallini CA, Michaud M, et al. Fusobacterium nucleatum potentiates intestinal tumorigenesis and modulates the tumor-immune microenvironment. Cell host \& microbe. 2013;14(2):207 - 15.

8. 8. Yang Y, Weng W, Peng J, Hong L, Yang L, Toiyama Y, Gao R, Liu M, Yin M, Pan C, Li H, Guo B, Zhu Q, Wei Q, Moyer MP, Wang P, Cai S, Goel A, Qin H, Ma Y. Fusobacterium nucleatum Increases Proliferation of Colorectal Cancer Cells and Tumor Development in Mice by Activating Toll-Like Receptor 4 Signaling to Nuclear Factor-kB, and Up-regulating Expression of MicroRNA-21. Gastroenterology. 2017 Mar;152(4):851-866.e24.

9. 9. Wu J, Li K, Peng W, Li H, Li Q, Wang X, et al. Autoinducer-2 of Fusobacterium nucleatum promotes macrophage M1 polarization via TNFSF9/IL-1 $\beta$ signaling. International immunopharmacology. 2019;74:105724.

10. 10. Yu T, Guo F, Yu Y, Sun T, Ma D, Han J, Qian Y, Kryczek I, Sun D, Nagarsheth N, Chen Y, Chen H, Hong J, Zou W, Fang JY. Fusobacterium nucleatum Promotes Chemoresistance to Colorectal Cancer by Modulating Autophagy. Cell. 2017 Jul 27;170(3):548-563.e16.

11. 11. Kolenbrander PE, Palmer RJ, Periasamy S, Jakubovics NS. Oral multispecies biofilm development and the key role of cell-cell distance. Nature Reviews Microbiology. 2010;8(7):471 - 80.

12. 12. Han YW, Shi W, Huang GT-J, Haake SK, Park N-H, Kuramitsu H, et al. Interactions between periodontal bacteria and human oral epithelial cells: Fusobacterium nucleatum adheres to and 
invades epithelial cells. Infection and immunity. 2000;68(6):3140-6.

13. 13. Alvarez AF, Barba-Ostria $C$, Silva-Jiménez $H$, Georgellis D. Organization and mode of action of two component system signaling circuits from the various kingdoms of life. Environmental microbiology. 2016;18(10):3210-26.

14. 14. Tierney AR, Rather PN. Roles of two-component regulatory systems in antibiotic resistance. Future microbiology. 2019;14(6):533 - 52.

15. 15. Mattos-Graner RO, Duncan MJ. Two-component signal transduction systems in oral bacteria. Journal of oral microbiology. 2017;9(1):1400858.

16. 16. Li X, Lv X, Lin Y, Zhen J, Ruan C, Duan W, et al. Role of two-component regulatory systems in intracellular survival of Mycobacterium tuberculosis. Journal of cellular biochemistry. 2019;120(8):12197-207.

17. 17. Zschiedrich $\mathrm{CP}$, Keidel V, Szurmant $\mathrm{H}$. Molecular mechanisms of two-component signal transduction. Journal of molecular biology. 2016;428(19):3752-75.

18. 18. Jacob-Dubuisson F, Mechaly A, Betton J-M, Antoine R. Structural insights into the signalling mechanisms of two-component systems. Nature Reviews Microbiology. 2018;16(10):585 - 93.

19. 19. Sidote DJ, Barbieri CM, Wu T, Stock AM. Structure of the Staphylococcus aureus AgrA LytTR domain bound to DNA reveals a beta fold with an unusual mode of binding. Structure. 2008;16(5):727 - 35.

20. 20. Sun F, Li C, Jeong D, Sohn C, He C, Bae T. In the Staphylococcus aureus two-component system sae, the response regulator SaeR binds to a direct repeat sequence and DNA binding requires phosphorylation by the sensor kinase SaeS. Journal of bacteriology. 2010;192(8):2111-27.

21. 21. Fan R, Shi X, Guo B, Zhao J, Liu J, Quan C, et al. The effects of L-arginine on protein stability and DNA binding ability of SaeR, a transcription factor in Staphylococcus aureus. Protein Expression and Purification. 2021;177:105765.

22. 22. Rooks MG, Veiga P, Reeves AZ, Lavoie S, Yasuda $K$, Asano $Y$, et al. QseC inhibition as an antivirulence approach for colitis-associated bacteria. Proceedings of the National Academy of Sciences. 2017;114(1):142-7.

23. 23. Massmig M, Reijerse E, Krausze J, Laurich C, Lubitz W, Jahn D, et al. Carnitine metabolism in the human gut: characterization of the two-component carnitine monooxygenase CntAB from Acinetobacter baumannii. Journal of Biological Chemistry. 2020;295(37):13065-78.

24. 24. Chen J, Ma M, Uzal FA, McClane BA. Host cell-induced signaling causes Clostridium perfringens to upregulate production of toxins important for intestinal infections. Gut Microbes. 2014;5(1):96107.

25. 25. Goto R, Miki T, Nakamura N, Fujimoto M, Okada N. Salmonella Typhimurium PagP-and UgtLdependent resistance to antimicrobial peptides contributes to the gut colonization. PloS one. 2017;12(12):e0190095.

26. 26. Wakimoto $S$, Nakayama-Imaohji $H$, Ichimura $M$, Morita $H$, Hirakawa $H$, Hayashi $T$, et al. PhoB regulates the survival of Bacteroides fragilis in peritoneal abscesses. PloS one. 2013;8(1):e53829. 
27. 27. van der Stel AX, van Mourik A, Heijmen-van Dijk L, Parker CT, Kelly DJ, van de Lest CH, et al. The $C$ ampylobacter jejuni RacRS system regulates fumarate utilization in a low oxygen environment. Environmental microbiology. 2015;17(4):1049-64.

28. 28. Giannakopoulou N, Mendis N, Zhu L, Gruenheid S, Faucher SP, Le Moual H. The virulence effect of CpxRA in Citrobacter rodentium is independent of the auxiliary proteins NlpE and CpxP. Frontiers in cellular and infection microbiology. 2018;8:320.

29. 29. Pacheco AR, Curtis MM, Ritchie JM, Munera D, Waldor MK, Moreira CG, et al. Fucose sensing regulates bacterial intestinal colonization. Nature. 2012;492(7427):113-7.

30. 30. Fujimoto M, Goto R, Haneda T, Okada N, Miki T. Salmonella enterica serovar Typhimurium CpxRA two-component system contributes to gut colonization in salmonella-induced colitis. Infection and immunity. 2018;86(7).

31. 31. Liu M, Hao G, Li Z, Zhou Y, Garcia-Sillas R, Li J, Wang H, Kan B, Zhu J. CitAB Two-Component System-Regulated Citrate Utilization Contributes to Vibrio cholerae Competitiveness with the Gut Microbiota. Infect Immun. 2019 Feb 21;87(3):e00746-18.

32. 32. Sonnenburg ED, Sonnenburg JL, Manchester JK, Hansen EE, Chiang HC, Gordon JI. A hybrid twocomponent system protein of a prominent human gut symbiont couples glycan sensing in vivo to carbohydrate metabolism. Proceedings of the National Academy of Sciences. 2006;103(23):8834-9.

33. 33. Lowe EC, Baslé A, Czjzek M, Firbank SJ, Bolam DN. A scissor blade-like closing mechanism implicated in transmembrane signaling in a Bacteroides hybrid two-component system. Proceedings of the National Academy of Sciences. 2012;109(19):7298 - 303.

34. 34. Hirakawa H, Kurushima J, Hashimoto Y, Tomita H. Progress Overview of Bacterial TwoComponent Regulatory Systems as Potential Targets for Antimicrobial Chemotherapy. Antibiotics (Basel). 2020 Sep 23;9(10):635.

35. 35. Rosales-Hurtado M, Meffre P, Szurmant H, Benfodda Z. Synthesis of histidine kinase inhibitors and their biological properties. Medicinal research reviews. 2020;40(4):1440-95.

36. 36. Gumerov VM, Ortega DR, Adebali O, Ulrich LE, Zhulin IB. MiST 3.0: an updated microbial signal transduction database with an emphasis on chemosensory systems. Nucleic acids research. 2020;48(D1):D459-D64.

37. 37. Robert X, Gouet P. Deciphering key features in protein structures with the new ENDscript server. Nucleic acids research. 2014;42(W1):W320-W4.

38. 38. Letunic I, Khedkar S, Bork P. SMART: recent updates, new developments and status in 2020. Nucleic Acids Research. 2021;49(D1):D458-D60.

39. 39. Kelley LA, Mezulis S, Yates CM, Wass MN, Sternberg MJ. The Phyre2 web portal for protein modeling, prediction and analysis. Nature protocols. 2015;10(6):845 - 58.

40. 40. Unger T, Jacobovitch Y, Dantes A, Bernheim R, Peleg Y. Applications of the Restriction Free (RF) cloning procedure for molecular manipulations and protein expression. J Struct Biol. 2010 Oct;172(1):34-44. 
41. 41. Zhang C, Li A, Wang R, Cao Y, Jiang H, Ouyang S, et al. Recombinant expression, purification and bioactivity characterization of extracellular domain of human tumor necrosis factor receptor 1 . Protein expression and purification. 2019;155:21 - 6 .

42. 42. Mueller AM, Breitsprecher D, Duhr S, Baaske P, Schubert T, Längst G. Microscale thermophoresis: a rapid and precise method to quantify protein-nucleic acid interactions in solution. Methods Mol Biol. 2017;1654:151-164.

43. 43. Walker JN, Crosby HA, Spaulding AR, Salgado-Pabón W, Malone CL, Rosenthal CB, et al. The Staphylococcus aureus ArlRS two-component system is a novel regulator of agglutination and pathogenesis. PLoS Pathog. 2013;9(12):e1003819.

44. 44. Fournier B, Hooper DC. A new two-component regulatory system involved in adhesion, autolysis, and extracellular proteolytic activity ofStaphylococcus aureus. Journal of bacteriology. 2000;182(14):3955-64.

45. 45. Nguyen M-P, Yoon J-M, Cho M-H, Lee S-W. Prokaryotic 2-component systems and the OmpR/PhoB superfamily. Canadian journal of microbiology. 2015;61(11):799-810.

46. 46. Bai J, Zhu X, Zhao K, Yan Y, Xu T, Wang J, et al. The role of ArlRS in regulating oxacillin susceptibility in methicillin-resistant Staphylococcus aureus indicates it is a potential target for antimicrobial resistance breakers. Emerging microbes \& infections. 2019;8(1):503 - 15.

47. 47. Radin JN, Kelliher JL, Párraga Solórzano PK, Kehl-Fie TE. The two-component system ArIRS and alterations in metabolism enable Staphylococcus aureus to resist calprotectin-induced manganese starvation. PLoS pathogens. 2016;12(11):e1006040.

48. 48. Hayat SM, Farahani N, Golichenari B, Sahebkar A. Recombinant protein expression in Escherichia coli (E. coli): what we need to know. Current pharmaceutical design. 2018;24(6):718 - 25.

49. 49. Casali N. Escherichia coli host strains. Methods Mol Biol. 2003;235:27-48.

50. 50. Kapatral V, Anderson I, Ivanova N, Reznik G, Los T, Lykidis A, et al. Genome sequence and analysis of the oral bacterium Fusobacterium nucleatum strain ATCC 25586. Journal of bacteriology. 2002;184(7):2005-18.

\section{Tables}

Due to technical limitations, table 1 is only available as a download in the Supplemental Files section.

\section{Figures}


a

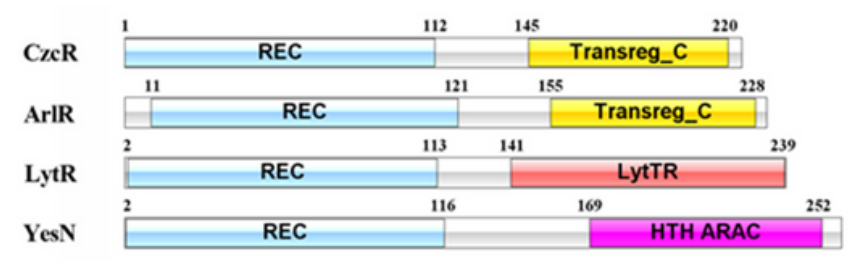

c

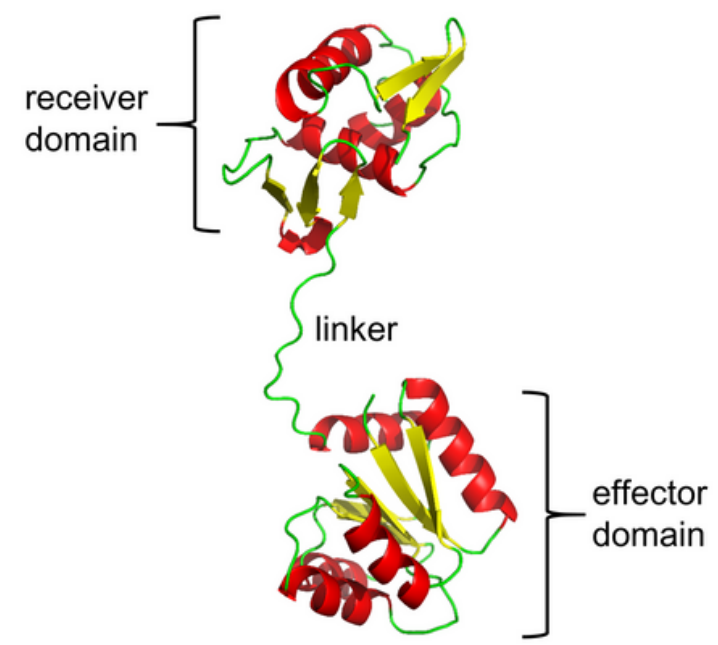

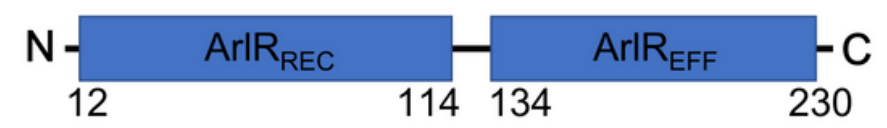

d
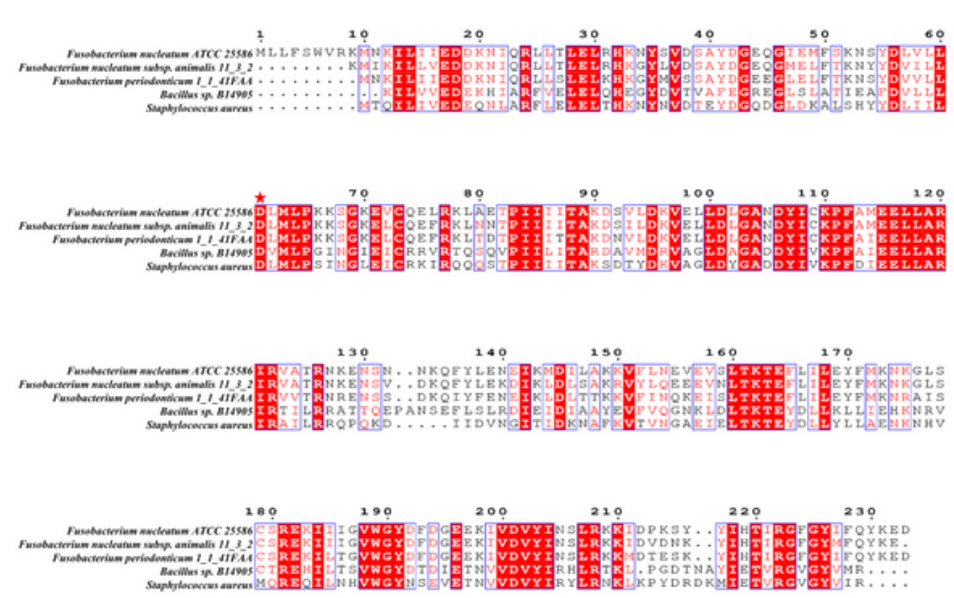

\section{Figure 1}

The molecular architecture of the two-component system response regulator protein and sequence alignment of ArlR protein in Fusobacterium nucleatum strain ATCC 25586 with ArlR in other strains. (a) The molecular architecture of the two-component system response regulator protein in Fusobacterium nucleatum ATCC 25586 predicted from SMART. REC: receiver domain; Transreg_C: transcriptional regulatory protein C-terminal; LytTR: LytTr DNA-binding domain; HTH_ARAC: helix-turn-helix arabinose operon control protein. (b) The molecular architecture of ArIR response regulator protein in Fusobacterium nucleatum strain ATCC 25586 predicted from Pfam. ArIRREC: ArIR receiver domain (from 12 to 114 th amino acid); ArIREFF: ArlR effector domain (from 134 to 230th amino acid). (c) The structure of ArlR response regulator protein in Fusobacterium nucleatum strain ATCC 25586 predicted from Phyre2. (d) Sequence alignment of ArlR protein in Fusobacterium nucleatum ATCC 25586 with ArlR protein in other strains. The conserved aspartic acid marked with a red asterisk is the predicted site receiving a phosphate group.

a

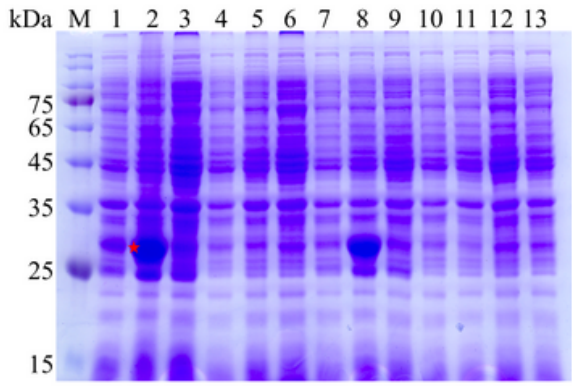

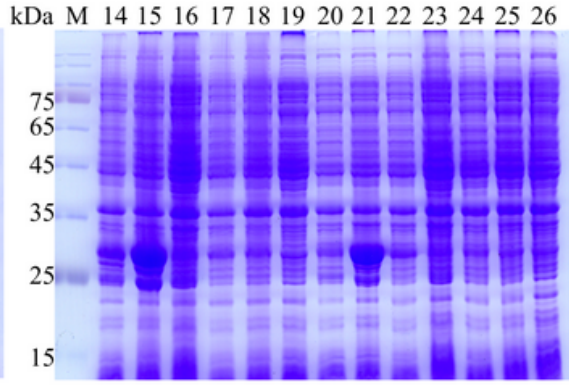

b

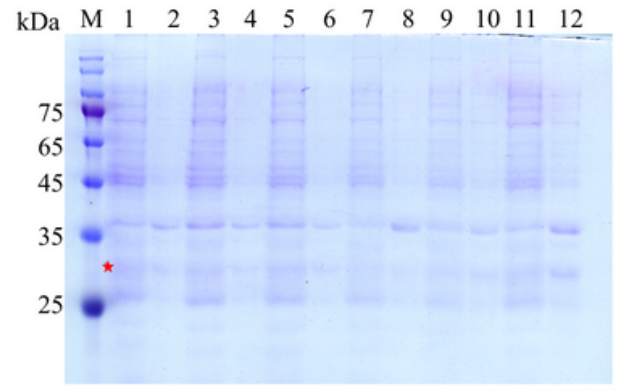


Optimizing of ArlR protein expression and purification conditions. (a) Screening of ArlR expression conditions in different host cells, culture temperature, and culture time: M-maker; 1 . Transetta, $25^{\circ} \mathrm{C}, 8 \mathrm{~h}$; 2. RIL, $25^{\circ} \mathrm{C}, 8$ h; 3. pLysS, $25^{\circ} \mathrm{C}, 8$ h; 4 . BL21, $25^{\circ} \mathrm{C}, 8$ h; 5 . Tuner, $25^{\circ} \mathrm{C}, 8$ h; $6 . \mathrm{C} 43,25^{\circ} \mathrm{C}, 8$ h; 7 .

Transetta, $16{ }^{\circ} \mathrm{C}, 8 \mathrm{~h} ; 8$. RIL, $16{ }^{\circ} \mathrm{C}, 8 \mathrm{~h} ; 9$. pLysS, $16{ }^{\circ} \mathrm{C}, 8 \mathrm{~h} ; 10$. BL21, $16{ }^{\circ} \mathrm{C}, 8 \mathrm{~h} ; 11$. Tuner, $16{ }^{\circ} \mathrm{C}, 8 \mathrm{~h} ; 12$. $\mathrm{C} 43,16{ }^{\circ} \mathrm{C}, 8 \mathrm{~h}$; 13. Control (BL21, $25^{\circ} \mathrm{C}, 8 \mathrm{~h}$, not induced); 14 . Transetta, $25^{\circ} \mathrm{C}, 20 \mathrm{~h} ; 15$. RIL, $25^{\circ} \mathrm{C}, 20 \mathrm{~h}$; 16. pLysS, $25^{\circ} \mathrm{C}, 20 \mathrm{~h} ; 17$. BL21, $25^{\circ} \mathrm{C}, 20 \mathrm{~h} ; 18$. Tuner, $25^{\circ} \mathrm{C}, 20 \mathrm{~h} ; 19 . \mathrm{C} 43,25^{\circ} \mathrm{C}, 20 \mathrm{~h} ; 20$. Transetta, 16 ${ }^{\circ} \mathrm{C}, 20$ h; 21. RIL, $16{ }^{\circ} \mathrm{C}, 20 \mathrm{~h} ; 22$. pLysS, $16{ }^{\circ} \mathrm{C}, 20$ h; 23. BL21, $16{ }^{\circ} \mathrm{C}, 20$ h; 24 . Tuner, $16{ }^{\circ} \mathrm{C}, 20 \mathrm{~h} ; 25$. C43, $16^{\circ} \mathrm{C}, 20 \mathrm{~h} ; 26$. Control (BL21, $25^{\circ} \mathrm{C}, 20 \mathrm{~h}$, not induced). The best expression conditions for ArlR are marked by a red asterisk. (b) Optimizing of ArlR purification conditions in buffers with different additives: M-maker; 1-2. $20 \mathrm{mM}$ Tris-HCl, $150 \mathrm{mM} \mathrm{NaCl}, 5 \%$ glycerol; 3-4. $20 \mathrm{mM}$ Tris-HCl, $150 \mathrm{mM} \mathrm{NaCl}, 50 \mathrm{mM} \mathrm{L-}$ Arg; 5-6. $20 \mathrm{mM}$ Tris-HCl, $150 \mathrm{mM} \mathrm{NaCl}, 1 \%$ Triton X-100; 7-8. $20 \mathrm{mM}$ Tris-HCl, $150 \mathrm{mM} \mathrm{NaCl}, 50 \mathrm{mM}$ betaine; 9-10. $20 \mathrm{mM}$ Tris-HCl, $150 \mathrm{mM} \mathrm{NaCl}, 50 \mathrm{mM} \mathrm{L-Arg}$ and $50 \mathrm{mM} \mathrm{L-Glu;} \mathrm{11-12.} 20 \mathrm{mM}$ Tris-HCl, $150 \mathrm{mM} \mathrm{NaCl} ; 1,3,5,7,9,11$. Supernatant protein obtained after ultrasonication and centrifugation; $2,4,6$, $8,10,12$. Precipitate protein obtained after ultrasonication and centrifugation. The best purification conditions for ArlR are marked by a red asterisk.

a
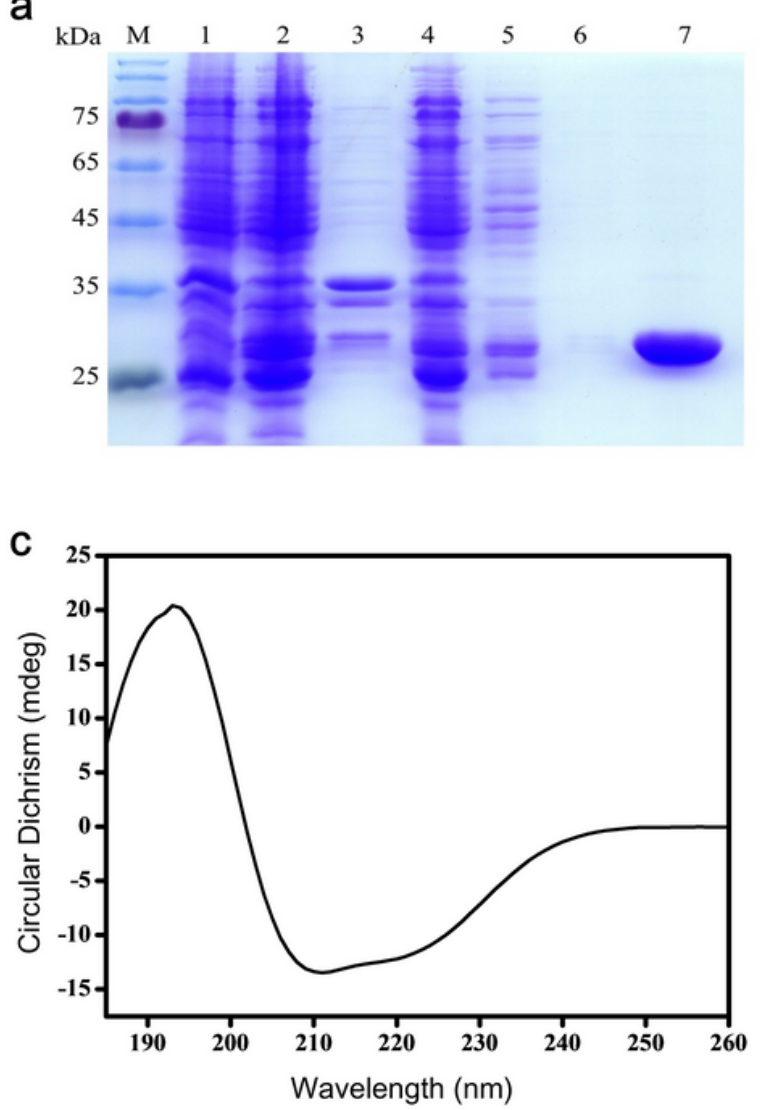

b

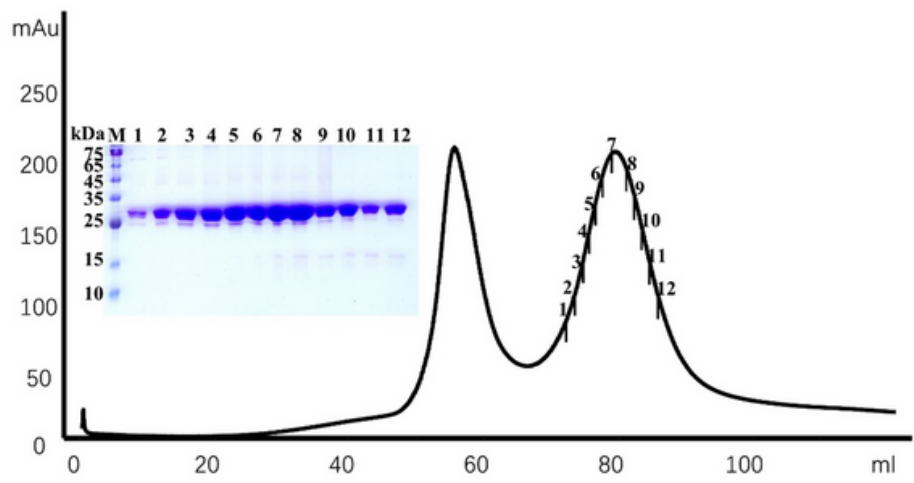

d

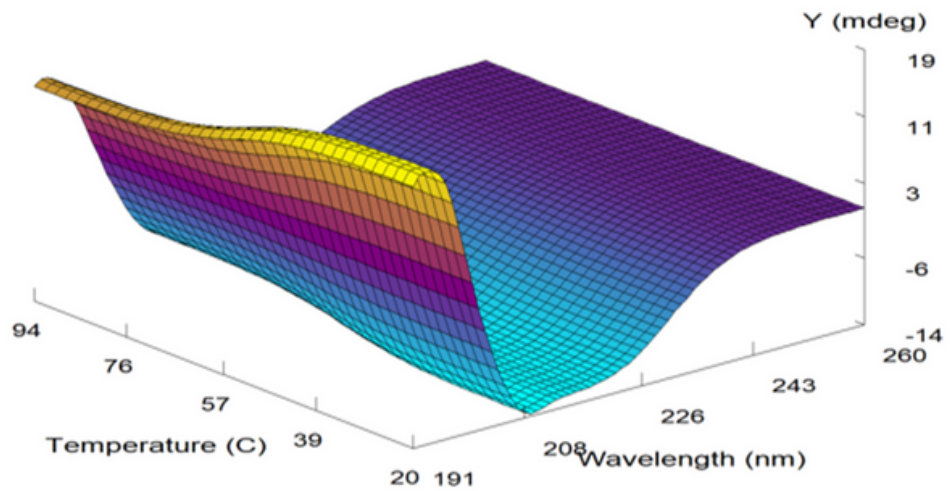

\section{Figure 3}

The secondary structure and thermal stability of the purified ArIR protein. (a) SDS-PAGE analysis of the expression and purification of ArIR: M-maker; 1. Protein after ultrasonication; 2. Supernatant protein after ultrasonication and centrifugation; 3. Precipitant protein after ultrasonication and centrifugation; 4. 
Unbound protein on nickel column; 5-6. Washing buffer eluted protein; 7. Elution buffer eluted protein (b) SDS-PAGE analysis of the ArIR purified by gel filtration chromatography. M: maker; the numbers 1-12 correspond to the fractions on the chromatogram. (c) The circular dichroism spectrum of the purified ArlR protein. (d) Circular dichroism spectrum of purified ArlR at varying temperatures from $20^{\circ} \mathrm{C}$ to $94{ }^{\circ} \mathrm{C}$.

a

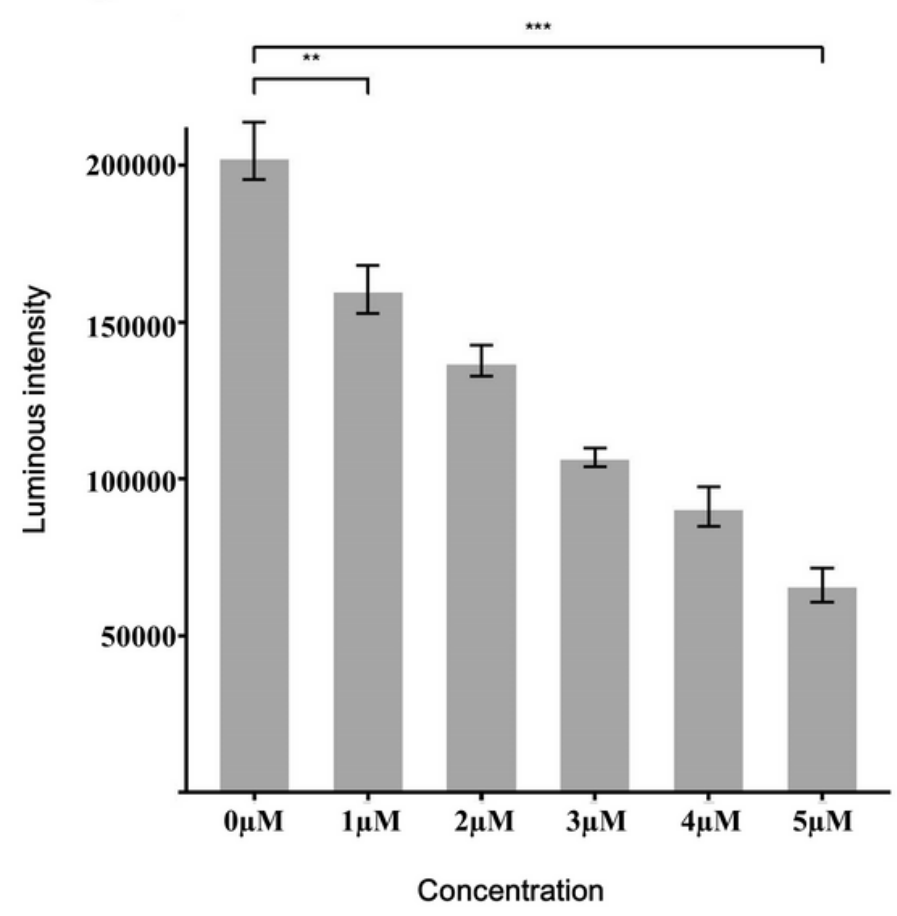

b

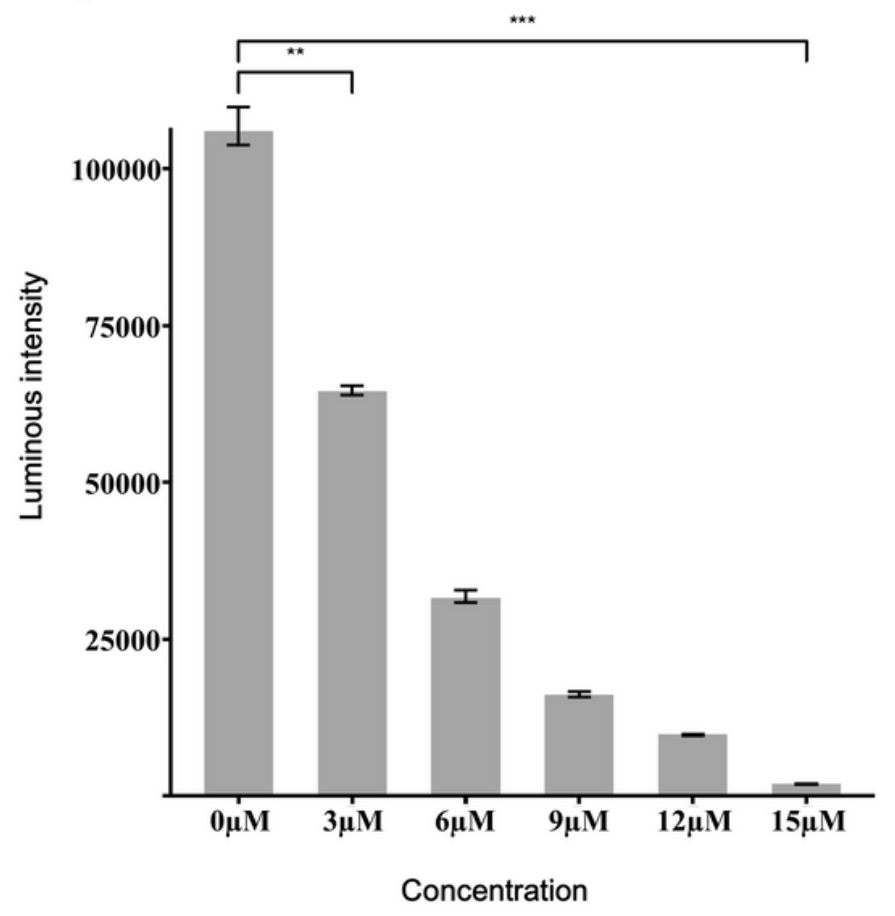

\section{Figure 4}

The kinase activity of ArISC and the phosphotransferase activity of P-ArISC to ArIR. ATP as a substrate by the Ultra-Glo ${ }^{\mathrm{TM}}$ Luciferase to catalyze producing photon of light. Hence, Luminescence intensity is directly proportional to the amount of ATP persisting in the reaction solution and inversely proportional to histidine-kinase activity or phosphotransferase activity. (a) The luminescence intensity of kinase reaction buffer. As the ArISC protein concentration gradually increases, the luminescence intensity of the buffer gradually decreases, i.e., the remaining ATP content in the reaction solution gradually decreases, indicating that the ArISC protein can bind ATP and has histidine-kinase activity. (b) The luminescence intensity of phosphotransferase reaction buffer. As the ArlR protein concentration gradually increases, the luminescence intensity gradually decreases, i.e., the remaining ATP content in the reaction solution gradually decreases, indicating that the P-ArISC protein can transfer phosphate group to ArlR. The experiment was repeated three times. Error bars represent average difference between the data points and their mean. $* *, p<0.01, * \star *, p<0.001$. 

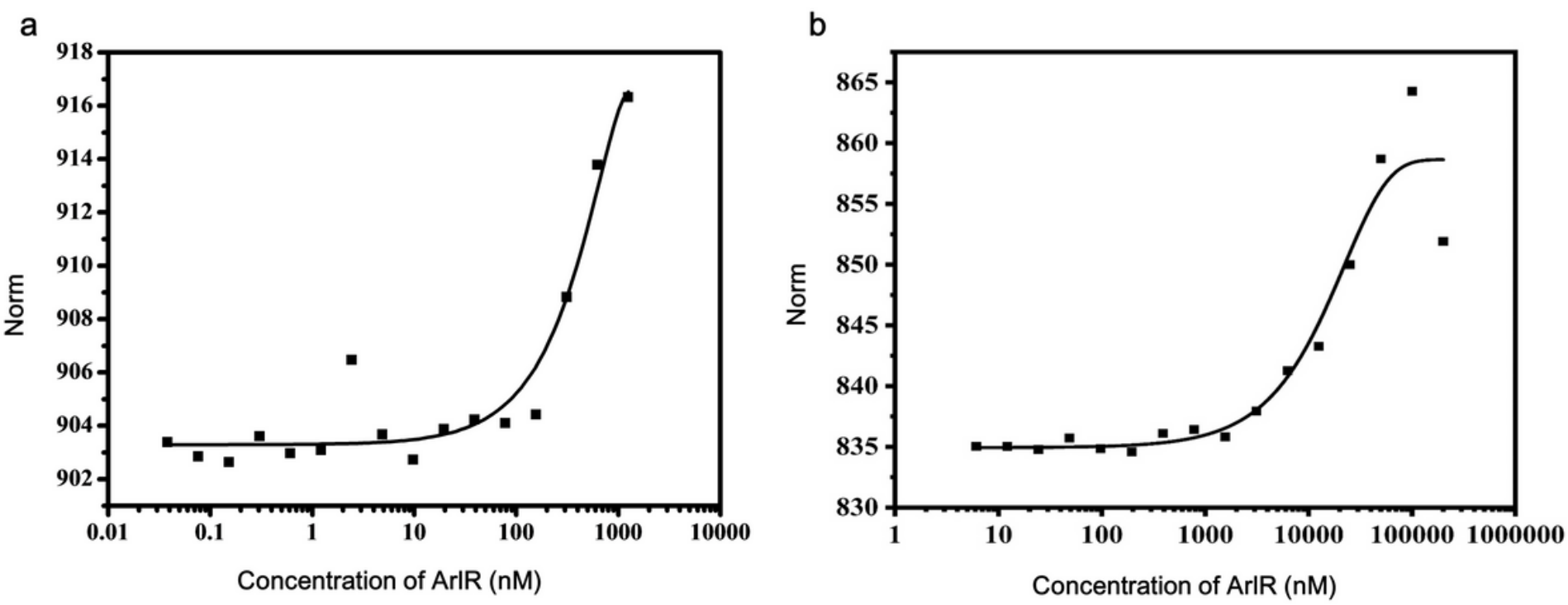

Figure 5

The interaction between P-ArlSC and its corresponding response regulator protein ArIR, and binding of PArlR to its promoter measured by MST assay. (a) MST showing the interaction between P-ArISC and ArIR with $\mathrm{Kd}=1.28 \mu \mathrm{M}$. (b) MST showing the binding of P-ArlR to its own promoter with $\mathrm{Kd}=37.5 \mu \mathrm{M}$.

\section{Supplementary Files}

This is a list of supplementary files associated with this preprint. Click to download.

- Table1.tiff.tif 